\title{
Black hole masses from X-rays
}

\author{
Xin-Lin Zhou ${ }^{1}$, Roberto Soria ${ }^{2}$ \\ ${ }^{1}$ National Astronomical Observatories, Chinese Academy of Sciences, \\ Beijing 100012, China \\ email: zhouxl@nao.cas.cn \\ ${ }^{2}$ International Centre for Radio Astronomy Research, Curtin University \\ GPO Box U1987, Perth, WA 6845, Australia \\ email: roberto.soria@icrar.org
}

\begin{abstract}
We discuss two methods to estimate black hole (BH) masses using X-ray data only: from the X-ray variability amplitude and from the photon index $\Gamma$. The first method is based on the anti-correlation between $\mathrm{BH}$ mass and X-ray variability amplitude. Using a sample of AGN with $\mathrm{BH}$ masses from reverberation mapping, we show that this method shows small intrinsic scatter. The second method is based on the correlation between $\Gamma$ and both the Eddington ratio $L_{\mathrm{bol}} / L_{\mathrm{Edd}}$ and the bolometric correction $L_{\mathrm{bol}} / L_{2-10 \mathrm{keV}}$.
\end{abstract}

Keywords. Accretion, accretion disks, X-rays:binaries, X-rays: galaxies

\section{X-ray variability amplitude}

The XVA $\sigma_{\text {rms }}^{2}$ (also known as "excess variance") is the variance of a light curve normalized by its mean squared after correcting for experimental noise (Nandra et al. 1997; Turner et al. 1999). For a light-curve segment with $N$ bins:

$$
\sigma_{\mathrm{rms}}^{2}=\frac{1}{N \mu^{2}} \sum_{i=1}^{N}\left[\left(X_{i}-\mu\right)^{2}-\sigma_{i}^{2}\right],
$$

where $X_{i}$ and $\sigma_{i}$ are count rates and uncertainties in each bin, and $\mu$ is the average of the count rates. There is an empirical (anti)correlation between XVA and BH masses in AGN ( $\mathrm{Lu} \& \mathrm{Yu} 2001$, O'Neill et al. 2005). To constrain this correlation, we selected and studied two (largely overlapping) AGN samples with X-ray observations longer than $40 \mathrm{ks}$ : one sample with $\mathrm{BH}$ masses derived from reverberation mapping, and the other from the $M_{\mathrm{BH}}-\sigma_{*}$ relation (see Zhou et al. 2010 for details). We found that the intrinsic dispersion of the $M_{\mathrm{BH}}-\sigma_{\mathrm{rms}}^{2}$ relation for the reverberation-mapped AGN is quite small, no larger than the uncertainties in the $\mathrm{BH}$ masses:

$$
M_{\mathrm{BH}}=10^{4.97 \pm 0.26}\left(\sigma_{\mathrm{rms}}^{2}\right)^{-1.00 \pm 0.10} M_{\odot} .
$$

A similar result was independently obtained by Ponti et al. (2012).

We used this relation to determine the BH mass in the Seyfert galaxies MCG-6-30-15 and 1H0707-495, using archival XMM-Newton data. We obtained BH masses of $(2.6 \pm$ $0.5) \times 10^{6} M_{\odot}$ and $(6.8 \pm 0.7) \times 10^{5} M_{\odot}$, respectively (Zhou et al. 2010). The XVA derived from multiple $X M M$-Newton observations changes by a factor of $2-3$. This means that the uncertainty in the $\mathrm{BH}$ mass from a single observation is slightly worse than that from the reverberation mapping or the stellar velocity dispersion methods. However, if the XVA randomly scatters around the true value for the power spectral density (PSD, Vaughan et al. 2003), the mean XVA of many data segments reduces the error. We conclude that the XVA might be a better BH mass estimator than the empirical virial method. 
The $M_{\mathrm{BH}}-\sigma_{\mathrm{rms}}^{2}$ relation is explained by a shift of the high-frequency break $f_{\mathrm{b}}$ in the $\mathrm{PSD}$, to lower frequencies for higher $\mathrm{BH}$ masses. $f_{\mathrm{b}}$ scales approximately as $\dot{m} / M_{\mathrm{BH}}$, where $\dot{m}$ is the dimensionless Eddington accretion rate (McHardy et al. 2006); however, we found that there is no or very weak correlation between XVA and $\dot{m}$, confirming the findings of O'Neill et al. (2005). This suggests that the normalization of the PSD varies with $\dot{m}$ in a way that compensates for the break-frequency dependence on $\dot{m}$.

Finally, we point out that our sample of AGN is skewed towards BHs with low mass and high Eddington rates. In forthcoming work, we will explore: where the relation saturates at the low-mass end (Ai et al. 2011); how it may extend to very-low-luminosity nuclear BHs in normal galaxies; and whether it may be used to estimate BH masses in ultraluminous X-ray sources (ULXs).

\section{BH mass estimates from $\Gamma$ and $L_{2-10 \mathrm{keV}}$}

X-ray spectral studies of accreting BHs show a correlation between the photon index $\Gamma$ of the power-law component and the Eddington ratio. Specifically, $\Gamma$ varies from $\approx 2.5$ for $L_{\mathrm{bol}} \sim L_{\mathrm{Edd}}$ to $\approx 1.5$ for $L_{\mathrm{bol}} \sim 10^{-2} L_{\mathrm{Edd}}$ (for AGN: Shemmer et al. $2008, \mathrm{Gu} \&$ Cao 2009; for stellar-mass BHs: Wu \& Gu 2008). (At even lower luminosities, there is evidence that $\Gamma$ increases again, but with a much weaker correlation: $\mathrm{Gu} \&$ Cao 2009, Corbel et al. 2008). In Zhou \& Zhao (2010), we refined this correlation by choosing a sample of 29 lowredshift $(z<0.33)$ AGN in the luminosity range $10^{-2} \sim L_{\mathrm{bol}} / L_{\mathrm{Edd}} \sim 1$. We determined $\Gamma$ by fitting their XMM-Newton/EPIC spectra in the $2-10 \mathrm{keV}$ range. We selected only radio-quiet AGN, because beaming in radio-loud sources may affect measurements of the intrinsic value of $\Gamma$. All sources have $\mathrm{BH}$ masses from reverberation mapping, and $L_{\mathrm{bol}}$ estimated from simultaneous X-ray, UV and optical observations. We obtain:

$$
\begin{gathered}
\log \left(L_{\mathrm{bol}} / L_{\mathrm{Edd}}\right)=(2.09 \pm 0.58) \Gamma-(4.98 \pm 1.04) \\
\log \left(L_{\mathrm{bol}} / L_{2-10 \mathrm{keV}}\right)=(1.12 \pm 0.30) \Gamma-(0.63 \pm 0.53)
\end{gathered}
$$

The correlation 2.2 appears stronger than 2.1 (see also Jin et al. 2012). In forthcoming work, we shall compare these correlations with those inferred from high-redshift AGN, to check for evolutionary effects in AGN spectral properties. Assuming no evolutionary effect, we can use $(2.1,2.2)$ to determine BH masses in AGN for which we know the $\mathrm{X}$-ray luminosity. We estimate a mean uncertainty in the $\mathrm{BH}$ mass of a factor of 2 or 3 (Shemmer et al. 2008). We shall also explore the $\Gamma$ versus $L_{\mathrm{bol}} / L_{\mathrm{Edd}}$ correlation for $L_{\mathrm{bol}} / L_{\mathrm{Edd}} \gtrsim 1$, with possible applications to ULXs and quasars.

\section{References}

Ai, Y. L., Yuan, W., Zhou, H. Y., Wang, T. G., \& Zhang, S. H. 2011, ApJ, 727, 31

Corbel, S., Körding, E., \& Kaaret, P. 2008, MNRAS, 389, 1697

Gu, M., \& Cao, X. 2009, MNRAS, 399, 349

Jin, C., Ward, M., \& Done, C. 2012, MNRAS, 425, 907

Lu, Y., \& Yu, Q. 2001, MNRAS, 324, 653

McHardy, I. M., Koerding, E., Knigge, C., Uttley, P., \& Fender, R. P. 2006, Nature, 444, 730

Nandra, K., George, I. M., Mushotzky, R. F., Turner, T. J., \& Yaqoob, T. 1997, ApJ, 476, 70

O’Neill, P. M., Nandra, K., Papadakis, I. E., \& Turner, T. J. 2005, MNRAS, 358, 1405

Ponti, G., et al. 2012, A\& $A, 542,83$

Shemmer, O., Brandt, W. N., Netzer, H., Maiolino, R., \& Kaspi, S. 2008, ApJ, 682, 81

Turner, T. J., George, I. M., Nandra, K., \& Turcan, D. 1999, ApJ, 524, 667

Vaughan, S., Edelson, R., Warwick, R. S., \& Uttley, P. 2003, MNRAS, 345, 1271

Wu, Q. \& Gu, M. 2008, ApJ, 682, 212

Zhou, X. L., Zhang, S. N., Wang, D. X., \& Zhu, L. 2010, ApJ, 710, 16

Zhou, X. L. \& Zhao, Y. H. 2010, ApJ, 720, L206 